\title{
A Method of Positioning Signal Transmission Based on Frequency Division Multiplexing
}

\author{
Zhongliang Deng ${ }^{1, a}$, Chao Dai ${ }^{2, b}$ \\ ${ }^{1}$ School of Electronic Engineering,Beijing University of Posts and Telecommunications,Beijing \\ 100876, China. \\ ${ }^{2}$ School of Electronic Engineering,Beijing University of Posts and Telecommunications,Beijing \\ 100876, China. \\ adengzhl@bupt.edu.cn, ${ }^{\mathrm{b}}$ daichao611@163.com
}

Keywords: signal transmission, positioning signal, frequency division multiplexing.

\begin{abstract}
With the development of mobile Internet, the demand for location based service highly increased. The widely used GNSS has a weakness for indoor positioning, while the Pseudolites Positioning System on the ground can achieve seamless outdoor and indoor positioning. Most of the Pseudolites Positioning System use the spread spectrum navigation and positioning signal system like GNSS, whose signal is usually generated followed by information modulation, spread spectrum modulation and RF modulation. Due to the specificity of indoor positioning, the system have to generate multiple signals carrying different information. To implement the indoor covering of positioning signal, every single signal should be wired to the indoor antenna individually, which increased the difficulty and cost of indoor covering. To reduce the difficulty of construction and solve the positioning accuracy problem caused by the differences of cables, the paper presents a method based on the frequency division multiplexing. The method transmits only one channel signal which is composed of multiple positioning signals assigned a different carrier frequency to multicarrier signal processing module used for choosing the specific positioning signal transmitted by specific antenna. The simulation result shows that the method can transmit multiple positioning signals by one cable to multiple antennas, reduce the cost of construction and improve the positioning accuracy at the same time.
\end{abstract}

\section{Introduction}

Recently, the location-based services and various types of applications based on it impact on people's lives. Global Navigation Satellite System (GNSS), represented by GPS has basically been able to meet the requirements of outdoor location-based services. But due to buildings, trees and other shelter and multipath effects, GPS and other GNSS systems performed poorly in the indoor positioning. The pseudolite positioning system use ground station to send positioning signals similar to a GPS positioning signal. Terminal receivers receive positioning signals and extract the pseudo-range, the signal time difference of arrival (TDOA, Time Difference of Arrival) and other relevant information, combined with inertial navigation can get more accurate indoor positioning results[1]. Indoor distribution system can be constructed similar to the mobile network to improve the coverage indoor positioning signal[2]. However, due to the special nature of the positioning signals, each positioning signals are to be transmitted via a separate cable from the transmitter to the indoor antenna, which increases the difficulty and cost of construction, limiting the development of pseudolite indoor positioning system. By researching the basic principles of positioning signal generation and receiving process, based on frequency division multiplexing and signal orthogonal ideas, we propose a method to transmit multi-channel positioning signals via a single cable. We introduced its theoretical basis and design methods and simulation to verify the feasibility of the method. 


\section{Signal Transmission over A Signal Cable}

The traditional indoor distribution system for mobile networks often use a main cable and divide the mobile communications signals to different indoor antenna through splitters. The design can meet the requirement of mobile communication and can significantly save costs of the project. Due to the special demand of the positioning, each antenna of the pseudo satellite positioning system which is equivalent to GPS satellite system to simulate a GPS system, need to send a different positioning signal[3]. Because of the increasing number of cable, the method would increase the complexity and cost of the construction. By analysis, the reason of this kind of design is needed because each indoor antenna requires transmit different signals. There comes a thought that if we transmit one channel of positioning signal composed by multi-channel signals via a single cable, then separating each positioning signals at the antenna by a particular method, then the traditional mobile network construction can be reused. Out of this simple idea, combining the theory of frequency division multiplexing and orthogonal signals, the method modulate different positioning signal to different frequency, then transmit the mixed signal over one cable. Then the re-adjusted corresponding signal which is restored through a particular band pass filter is transmitted on the antenna side. Based on the above analysis, the process of positioning signal generation and transmission can be can be described as shown in Figure1.

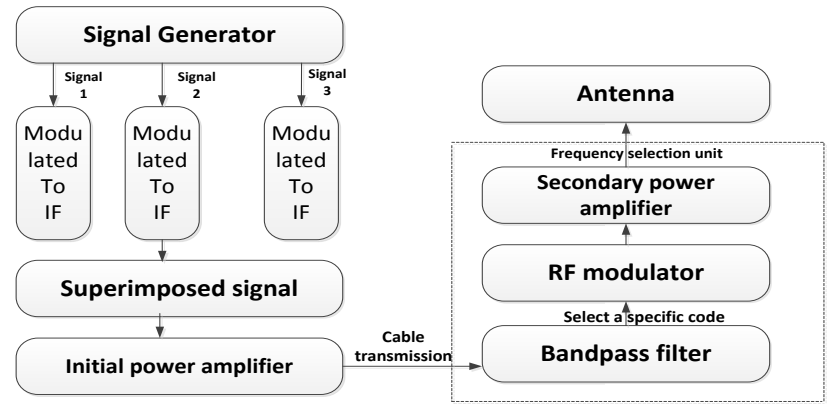

Fig. 1 Flow chart: Indoor positioning signal generation and transmission

\section{Theoretical Analysis}

The indoor base station generates the mixed signal transmitted over the cable which composed multiple different location codes modulated to the different intermediate frequency. The pseudolite positioning system usually use the Gold sequence as spreading sequence. Leaving aside the positioning signal modulated message information, the transmission signal on the cable can be expressed as:

$$
s(t)=A_{1} \sum_{i=0}^{N-1} x_{i}(t) e^{j 2 \pi\left(f_{1}+i^{*} \Delta f\right) t}
$$

In the equation, $s(t)$ is the signal generated by the signal generator, $N$ is the group number of positioning signals, $x_{i}(t)$ is the ith positioning signal, $\Delta f$ is the IF modulation frequency interval, and can be set to $0-100 \mathrm{MHz}$. When the signal reaches the positioning signal transmitter, the signal selection obtain the particular signal at corresponding frequency point via a frequency selective bandpass filter, and then transmit by the indoor antenna after second RF modulation and power amplification. The signal through the band-pass filter can be expressed as: $y(t)=s(t) \times h(t)$.In the equation, $h(t)$ is the impulse response of the filter. After decomposition of filter impulse response, the signal output from the antenna can eventually be reduced as:

$\mathrm{y}_{j}(t)=A_{1} A_{2} \sum_{i=0}^{N-1} \alpha_{i j} x_{i}\left(t-t_{i}\right) e^{j 2 \pi\left(f_{l}+i^{*} \Delta f\right)-\theta_{i}} * e^{j 2 \pi\left(f_{F}-f_{1}\right) t}$

In the equation, $A_{1}$ is the first amplification factor, $A_{2}$ is the product of the secondary amplification factor and the amplitude attenuation coefficient, $\alpha_{i j}$ is the attenuation factor of the band-pass filter with different frequencies, $f_{F}$ is the RF carrier frequency. Because of the nonlinear 
and non-ideal characteristics of the filter, the presence of residual carrier phase offset and other indoor location signals, positioning results will be affected. Below we will use the theoretical analysis and simulation demonstrate that using a particular intermediate frequency interval, with an improved carrier tracking loop can significantly reduce the residual positioning symbol interference.

\subsection{Selection the Frequency Interval.}

As is shown in Figure 1, the method of transmitting multi-channel signal over a single cable is to modulate different positioning signal to different frequency, then transmit the mixed signal over one cable. Then the re-adjusted corresponding signal which is restored through a particular band pass filter is transmitted on the antenna side. In this method, the selection of frequency internal is the most important. If the frequency interval is set too high, it will increase the burden on the receiver RF front-end and if the frequency interval is set too small, band-pass filter can't filter out other positioning signal frequency, resulting in residual positioning signal interference. By taking thought of signals orthogonal in a multi-carrier communication systems such as orthogonal frequency division multiplexing (OFDM, Orthogonal frequency-division multiplexing) system,[4]

$\int_{0}^{T} e^{j 2 \pi f_{n} t} * e^{j 2 \pi f_{m} t} d t=T e^{j \pi\left(f_{n}-f_{m}\right) T} \operatorname{sinc}\left[\left(f_{n}-f_{m}\right) T\right]$

when $\Delta f=\left|f_{n}-f_{m}\right|=1 / T$, two signals are orthogonal. According to this theory, the frequency interval $\Delta f$ is set equal to the code frequency $1 / T$. Frequency interval may be set such that the intermediate frequency and the code frequency are in the same order of magnitude, can reduce the performance requirements of the filter, reducing construction costs. At the same time when the interval frequency is equal to the code frequency, any two mutually positioning signals are orthogonal. The following paragraphs explain the advantages of orthogonal signals so that the receiving process will further reduce the residual positioning signal interference.

\subsection{Affect Caused by the Transmission of Signal.}

Due to non-ideal characteristics of the filter, the positioning signal which output from the antenna containing frequency components of the residual positioning code. Residual frequency of the residual positioning signal relatively close to the residual frequency of target positioning signal, so it may interferer receiver receiving target signal. The reception of the positioning signal compose the carrier tracking and stripping[5], the stripping process of the carrier frequency can be expressed as the following equation:

$$
r(t)=\int A \sum_{i=0}^{N-1} a_{i} X_{i}\left(t-t_{i}\right) e^{j 2 \pi\left(f_{t}+i^{*} \Delta f\right)-\theta_{i}} * e^{-j 2 \pi f_{r} t} d t
$$

In the equation, $A \sum_{i=0}^{N-1} a_{i} x_{i}\left(t-t_{i}\right) e^{j 2 \pi\left(f_{t}+i^{*} \Delta f\right)-\theta_{i}}$ is the intermediate frequency signal through the radio frequency front-end processing unit, $f_{I}$ represents the intermediate frequency of target signal. When tracking is steady, due to the carrier loop, $f_{r} \rightarrow f_{I}$. Suppose the channel NO.0 is the target positioning signal in the transmission signal , the remaining noise component can be expressed as[6]: $\mathrm{z}_{0}(t)=\int A \sum_{i=1}^{N-1} a_{i} x_{i}\left(t-t_{i}\right) e^{j 2 \pi i^{*} \Delta f-\theta_{i}} d t$

This noise may make the carrier tracking loop loses lock. In order to mitigate the effects of interference sources, make tracking stable, we proposed an improved carrier tracking loop , which is using a known carrier frequency to process the secondary carrier peel of the output signal, results in a ratio of the integral filter is input to the phase detector. Improved carrier tracking loop is shown in Figure 2. According to the theory of orthogonal signal and OFDM, when $\Delta f$ is set to the code rate $1 / T$, we can get the equation,

$n_{0}(t)=\frac{1}{T} \int_{0}^{T} z_{0}(t) * e^{j 2 \pi \Delta f} d t=\frac{1}{T} \int_{0}^{T} A \sum_{i=1}^{N-1} a_{i} x_{i}\left(t-t_{i}\right) e^{j 2 \pi i^{*} \Delta f-\theta_{i}} * e^{j 2 \pi \Delta f} d t=0$

From the above equation, the improved carrier tracking loop has no effect on normal tracking results. But this design can reduce the interference s for target acquisition and tracking, the carrier tracking loop can be improved to track target signal faster and more stable. 


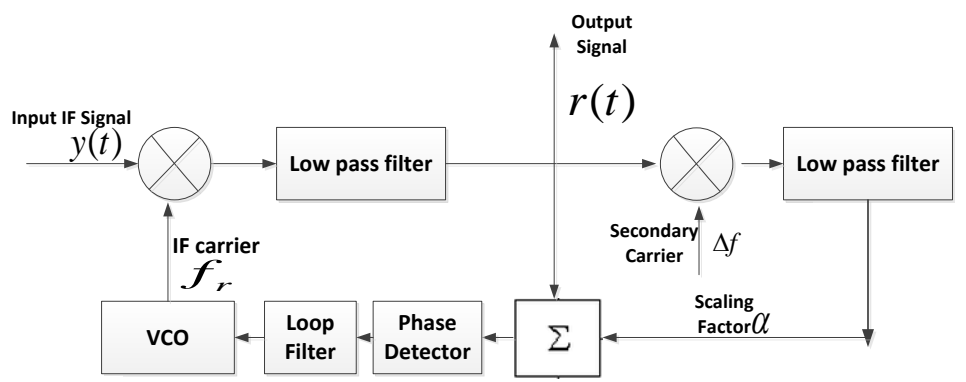

Fig. 2 Improved carrier tracking loop

\section{Demonstration and Simulation}

In order to verify the above theory, we use a spreading code which length is 8191 and rate is $5 \mathrm{MHz}$.We use three different positioning signal code 1 , code 2 , code 3 , which have the same average power, IF is $1 \mathrm{MHz} 、 6 \mathrm{MHz} 、 11 \mathrm{MHz}$ respectively. The default difference of TDOA is 50 between code 2 and code 3 . We use the Matlab to simulate the process of signal generation, transmission and reception described by Figure 1, verifying the results. By comparing pre-TDOA value and the TDOA value received. Figure 3 shows the process of receiving code 2, as can be seen, after 100 times of integration, receiver began tracking the code 2. Figure 4 shows the difference of TDOA between the code 2 and code 3 . The mean value of the TDOA shown in Figure 4 is -50.05 , close to the pre-set value of 50. That shows the use of the proposed method to generate and transmit signals, using an improved carrier tracking loop instead of the traditional tracking loop, can receive positioning signals efficiently, not affect the positioning result at the same time.

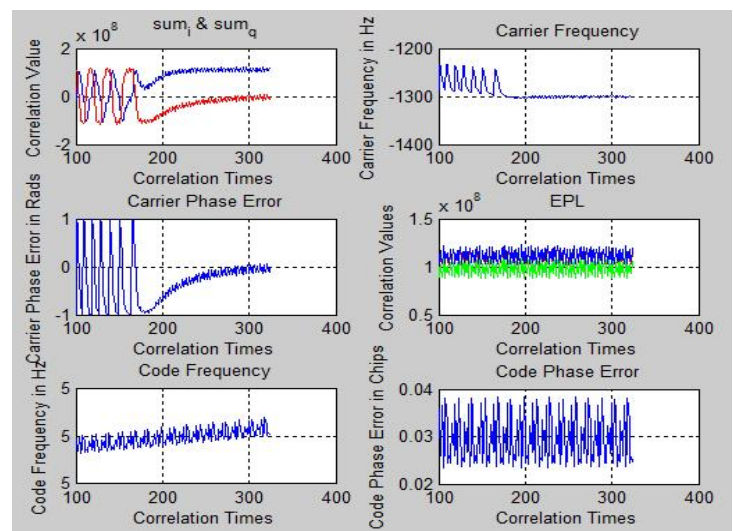

Fig. 3 Tracking of Code 2

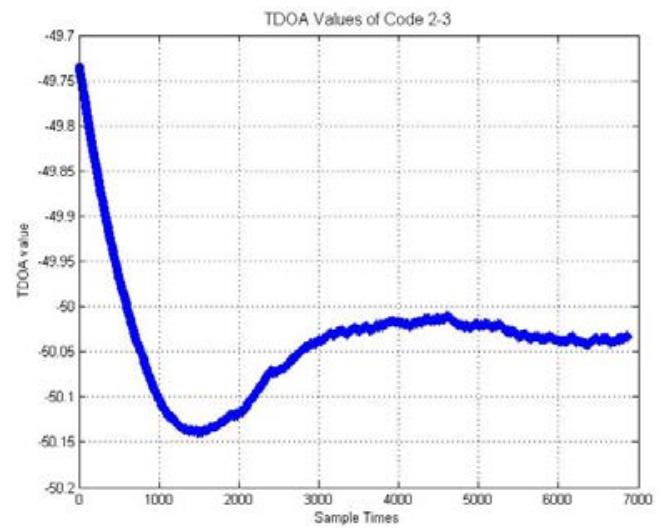

Fig. 4 TDOA of Code 2 \& Code 3

\section{Summary}

The results showed that the use of orthogonal frequency division multiplexing signals and the process of signal generation and transmission, using an improved carrier tracking loop instead of the traditional tracking loop, can achieve using a single cable to transmit multi-channel indoor positioning signals without affecting the positioning results. This method is used in engineering practice, can significantly reduce the complexity and cost of wiring, to promote the popularization and application of pseudolite indoor positioning system to a certain extent.

\section{References}

[1]. Zhang Yubao,Cui Xiaowei. A Design Scheme of Indoor Pseudolites Positioning System Based on Carrier Phase. Science Technology and Engineering. p. 6038-6042.

[2]. Wang Ying, Cheng Ritao, Zhang Haitao. TD-LTE planning and design methods study in indoor distributed antenna system. Telecom Engineering Technics and Standardization. p. 28-34. 
[3]. Peng Ruixue, Xu Lin,Wang Fu. Study of Configuration of Pseudo-Satellite Positioning System. Ordnance Industry Automation. 2010,08:53-56. p. 53-56.

[4]. Li Taohu, Liu Jiansheng, Huang Zhigang. A solution of the near-far effect based on the orthogonality of pseudolite signal . Electronics \& Information Technology . p. 1366-1369.

[5]. Hu Dan:Research of location and tracking algorithm cellular networks based on TDOA(Master,University of Electronic Science and Technology, China 2013).

[6]. Chai Junshuan,Zhang Xiaohui. Design and analyzing of carrier tracking loop for GPS receiver. Electronic Design Engineering. p. 118-121. 